${ }^{12}$ Emory University, Atlanta, GA, USA. ${ }^{13}$ Medstar Georgetown University Hospital, Washington, DC, USA. ${ }^{14}$ Hoag Memorial Hospital Presbyterian, Newport Beach, CA, USA. ${ }^{15}$ Logan Health Research Institute, Kalispell, MT, USA. ${ }^{16}$ Long Beach Medical Center, Long Beach, CA, USA. ${ }^{17}$ Lankenau Institute for Medical Research, Wynnewood, PA, USA. ${ }^{18}$ Montefiore Medical Center, New York, NY, USA. ${ }^{19}$ Albert Einstein College of Medicine, New York, NY, USA. ${ }^{20}$ Northwestern University Feinberg School of Medicine, Chicago, IL, USA. ${ }^{21}$ University of California Davis, Sacramento, CA, USA. ${ }^{22}$ University of California Irvine, Orange, CA, USA. ${ }^{23}$ University of Colorado, School of Medicine, Aurora, CO, USA. ${ }^{24}$ University of Pennsylvania, Philadelphia, PA, USA. ${ }^{25}$ Kaiser Permanente Southern California Physicians Group, Los Angeles, CA, USA. ${ }^{26}$ Keck School of Medicine, USC,
Los Angeles, CA, USA. ${ }^{27}$ University of Southern California, Los Angeles, CA, USA. ${ }^{28}$ Yale School of Medicine, New Haven, CT, USA.

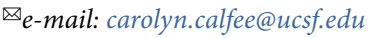

Published online: 20 January 2022

https://doi.org/10.1038/s41591-021-01617-x

\section{References}

1. RECOVERY Collaborative Group. et al. N. Engl. J. Med. 384, 693-704 (2020).

2. RECOVERY Collaborative Group. et al. Lancet 396, 1345-1352 (2020).

3. Abaleke, E. et al. Lancet 397, 605-612 (2021).

4. Angus, D. C. et al. Ann. Am. Thorac. Soc. 17, 879-891 (2020).

5. Writing Committee for the REMAP-CAP Investigators. JAMA 324, 1317-1329 (2020)

6. Barker, A. et al. Clin. Pharmacol. Ther. 86, 97-100 (2009).

7. Harrington, D. \& Parmigiani, G. N. Engl. J. Med. 375, 7-9 (2016)

8. Carey, L. A. \& Winer, E. P. N. Engl. J. Med. 375, 83-84 (2016).

9. Sinha, P. et al. Lancet Respir. Med. 8, 1209-1218 (2020).
Author contributions

All authors were involved in the conceptualization and writing and/or editing of this correspondence. L.E., C.C. K.D.L. and P.H. contributed to funding acquisition and L.E., C.C. and K.D.L. prepared the first draft.

\section{Competing interests}

L.E. is an unpaid member of the board of directors of Quantum Leap Healthcare Collaborative (QLHC, the study sponsor), and received grant funding from QLHC for the I-SPY TRIAL. L.E. is a member of the Blue Cross/Blue Shield Medical Advisory Panel and receives reimbursement for time and travel. L.E. has a grant from Merck for an Investigator-initiated trial of ductal carcinoma in situ. C.C. received funding for this article from QHLC, as well as other funding from the NIH and Roche/Genentech. C.C. also provides consulting services for Quark, Vasomune and Genle Life Sciences. K.D.L. is a stockholder of Amgen Inc. A.D..B is a member of the Scientific Advisory Board Committee of Caris Life Sciences. All other authors report no competing interests.

\title{
Understanding and tracking the impact of long COVID in the United Kingdom
}

To the Editor - There is now a rich body of knowledge on acute COVID-19, but much less is known about the risk factors, clinical presentation, duration and management of persistent or new symptoms following recovery from initial infection, often termed long COVID $^{1}$. Post-infection follow-up data show that a significant proportion of hospitalized and non-hospitalized patients experience persistent symptoms and organ dysfunction ${ }^{2-4}$.
The UK Office for National Statistics (ONS) estimates that the number of people in the UK that are self-reporting symptoms lasting more than 4 weeks currently stands at 1.1 million ( $1.7 \%$ of the population $)^{3}$, while the real-time assessment of community transmission (REACT) study in England estimated the overall number of people who reported at least one symptom lasting for 12 or more weeks as more than 2 million by February 2021 (ref. ${ }^{5}$ ). Patients globally have reported a range of new, returning and/or ongoing symptoms, including (but not limited to) fatigue, shortness of breath, altered smell and taste, cough, myalgia, cognitive impairment and diarrhea following COVID-19 infection ${ }^{6}$. Of great concern are data from imaging studies that report single or multiple organ impairment, even in non-hospitalized patients?

To build greater understanding of long COVID, a wide range of nationally funded studies has been launched in the UK (see Fig. 1 and Table 1). The post-hospitalisation

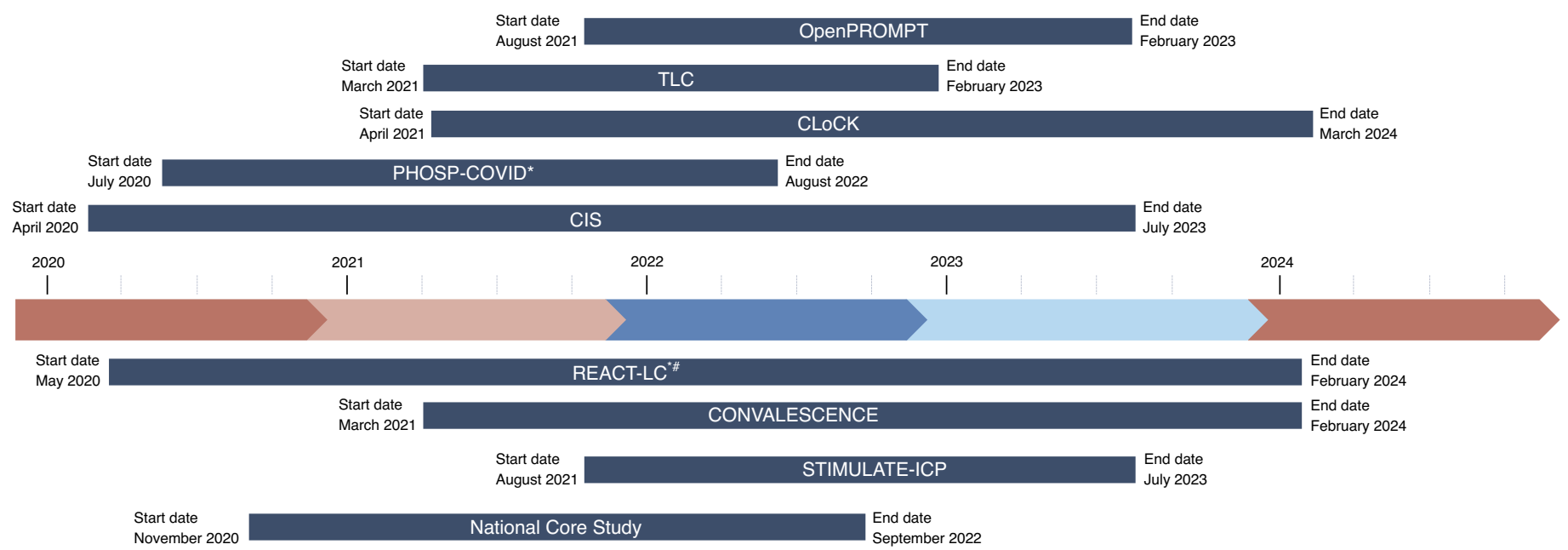

Fig. 1 | Timeline of major funded epidemiological studies on long COVID in the UK. *PHOSP-COVID has ethical approval for 25-year follow-up and REACT-LC for 20-year follow-up, with plans highlighting how each study will evolve, ask new questions and seek further resources. \#REACT-LC is funded from February 2021 but the timeline of the project starts in May 2020 with the use of the first baseline data from REACT participants, which form the sampling for follow-up. 


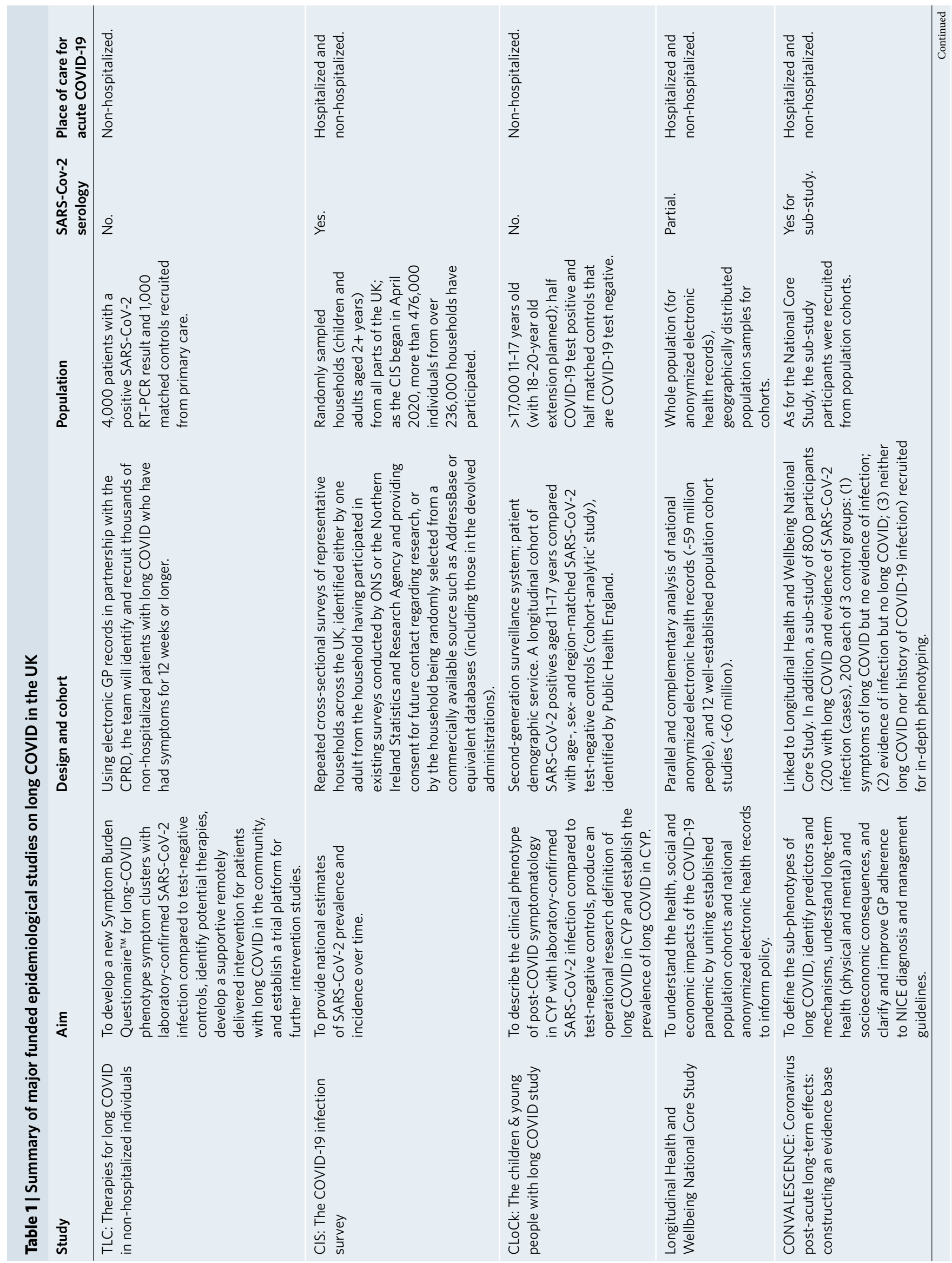




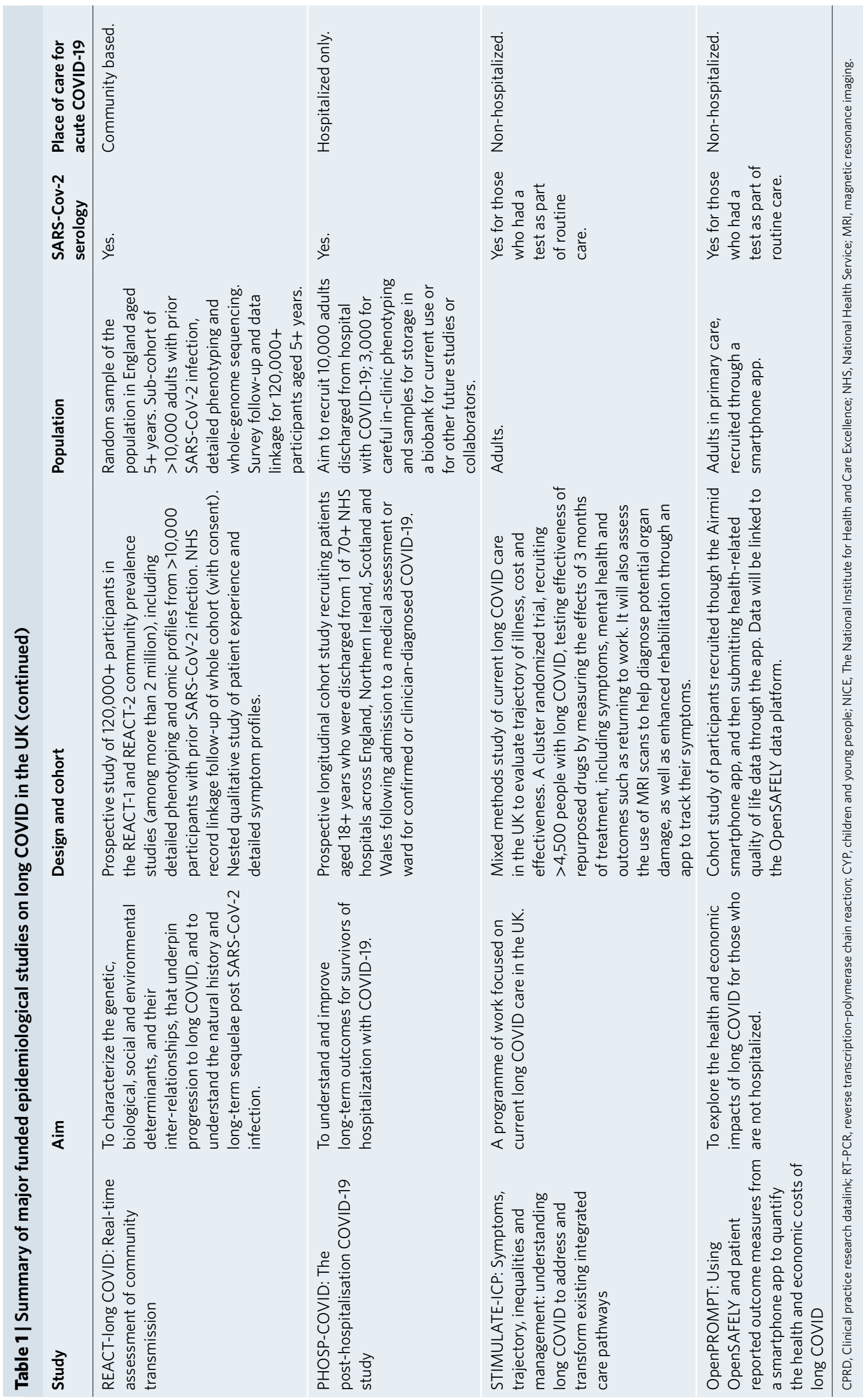


COVID-19 study (PHOSP-COVID) was the first such study, securing $£ 8.4$ million in July 2020 from UK Research and Innovation (UKRI), and aims to understand and improve long-term outcomes for people who survive hospitalization with COVID-19.

Another study that started early in the pandemic was the REACT programme, which was commissioned by the Department of Health and Social Care in April 2020. The REACT studies are nationally representative prevalence surveys of SARS-CoV-2 in the community in England. REACT was then extended to include REACT-long COVID (REACT-LC) in February 2021 via the National Institute for Health Research (NIHR) and UKRI funding. REACT-LC aims to characterize the genetic, biological, social and environmental determinants, and the corresponding inter-relationships, that underpin progression to long COVID, and to understand the natural history and long-term sequelae after SARS-CoV-2 infection. In early 2021, the NIHR and UKRI funded four research studies (totalling $£ 18.5$ million) to better understand and address longer-term effects of SARS-CoV-2 infection on physical and mental health. In summer 2021, NIHR funded a further 15 projects (totalling $£ 19.6$ million) to improve the diagnosis of long COVID, as well as treatment, rehabilitation and recovery.

From this cohort of funded projects, as well as other key national projects, nine major epidemiology-focused long COVID studies are currently running in the UK (see Fig. 1 and Table 1). These studies cover population epidemiology, symptomatology, phenotyping, operational definition, health impacts post-hospitalization, determinants, mechanisms and consequences of the long-term effects, and genetic, biological, social and environmental signatures and pathways. Researchers on these studies have formed the National Long COVID Research working group, to share key findings and promote rapid knowledge exchange and efficient timely research. Key outputs of the group are shared with the Chief Medical Officer for England. An example of this knowledge exchange in action is an open science repository platform ${ }^{8}$ that the National Long COVID working group has formed to collate and index the latest research on long COVID.

There are a wide range of outstanding research priorities, including defining long COVID, identifying its pathogenesis, describing its clinical presentation and health impacts, characterizing the longer-term burden and predictive risk factors and developing clinical interventions. The primary areas of knowledge that the studies outlined in Table 1 will advance are: the prevalence and phenotyping of symptom clusters (TLC, CIS, CONVALESCENCE, REACT-LC and PHOSP-COVID; see Table 1 for full study names), identification of the short- and long-term mental and physical health sequelae of long COVID (TLC, CIS, CLoCk, PHOSP-COVID, CONVALESCENCE, REACT-LC, STIMULATE-ICP and OpenPROMPT), prevalence, phenotyping and operational research definition of long COVID in children and young people (CLoCk), the immunological causes and consequences of long COVID (TLC and REACT-LC), identifying mechanisms that underpin the development of long COVID (TLC, CONVALESCENCE and REACT-LC), and identifying and trialling potential therapies and interventions to treat and manage long COVID (TLC, PHOSP-COVID and STIMULATE-ICP). The CLoCk study is the largest study of long COVID specifically in children that we know of globally, uniquely uses child-reported symptoms, a control group and standardized measures, and is longitudinal and based on a national cohort.

A particular strength of several of the studies (TLC, CLoCk, CONVALESCENCE and the REACT cohort follow-up) is the use of control groups, which allows comparison of symptom profiles and burden in individuals with confirmed SARS-CoV-2 infection compared to those without infection who may experience symptoms due to comorbidities, and because some reported long-COVID symptoms are non-specific and prevalent in the general population ${ }^{9}$. There is a huge burden of ill health from long COVID in the UK and globally. People living with long COVID were the first to identify and describe long $\mathrm{COVID}^{10}$. The research community have joined patient researchers in their efforts to improve understanding of long COVID, including its symptoms, health impacts, pathophysiology and treatments. Patient and public involvement is a feature of all the studies described above, with research teams working with patient partners from diverse backgrounds, as well as engaging with national long-COVID support groups. Multidisciplinary research collaboration with patients at the core, as well as co-producing the research as equal partners, is crucial for developing a comprehensive approach to unraveling the complexity and heterogeneity of long COVID. This co-production will be fundamental in the development of targeted therapeutic approaches and to inform health service provision to meet the large unmet need from long COVID.

Ash Routen', Lauren O'Mahoney (D)', Daniel Ayoubkhani², Amitava Banerjee (D)3
Chris Brightling ${ }^{4}$, Melanie Calvert (D) 5,6,7,8,9, Nishi Chaturvedi (D) ${ }^{10}$, Ian Diamond ${ }^{2}$, Rosalind Eggo ${ }^{11}$, Paul Elliott (D12, Rachael A. Evans (D) ${ }^{4}$, Shamil Haroon (D), Emily Herret ${ }^{11}$, Margaret E. O'Hara ${ }^{13}$, Roz Shafran (D) 14, Julie Stanborough², Terence Stephenson ${ }^{14}$, Jonathan Sterne (D) Helen Ward (D) 12 and Kamlesh Khunti ${ }^{1 \times}$ ${ }^{1}$ Diabetes Research Centre, Leicester General Hospital, University of Leicester, Leicester, UK. ${ }^{2}$ Office for National Statistics, Government Buildings, Newport, UK. ${ }^{3}$ Faculty of Population Health Sciences, Institute of Health Informatics, University College London, London, UK. ${ }^{4}$ Department of Respiratory Sciences, University of Leicester, Leicester, UK. ${ }^{5}$ Institute of Applied Health Research, University of Birmingham, Birmingham, UK. ${ }^{6}$ National Institute for Health Research Applied Research Centre West Midlands, Birmingham, UK. ${ }^{7}$ National Institute for Health Research Birmingham Biomedical Research Centre, University of Birmingham, Birmingham, UK. ${ }^{8}$ Birmingham Health Partners Centre for Regulatory Science and Innovation, University of Birmingham, Birmingham, UK. ${ }^{9}$ National Institute for Health Research Surgical Reconstruction and Microbiology Research Centre, University of Birmingham, Birmingham, UK. ${ }^{10}$ Department of Population Science and Experimental Medicine, University College London, London, UK. ${ }^{11}$ Department of Epidemiology and Population Health, London School of Hygiene and Tropical Medicine, London, UK. ${ }^{12}$ Faculty of Medicine, School of Public Health, Imperial College London, London, UK. ${ }^{13}$ Long Covid Support, Birmingham, UK. ${ }^{14}$ Great Ormond Street Institute of Child Health, University College London, London, UK. ${ }^{15}$ Population Health Sciences, Bristol Medical School, University of Bristol, Bristol, UK. $凶_{e-m a i l: k k 22 @ l e . a c . u k}$

Published online: 22 November 2021 https://doi.org/10.1038/s41591-021-01591-4

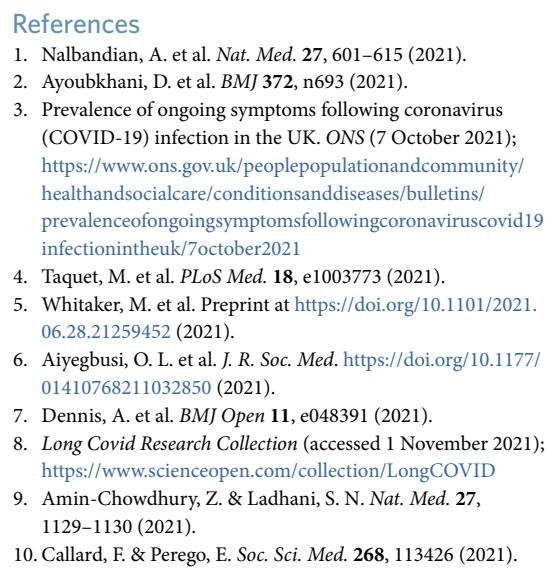

4. Taquet, M. et al. PLoS Med. 18, e1003773 (2021).

5. Whitaker, M. et al. Preprint at https://doi.org/10.1101/2021. 06.28.21259452 (2021).

6. Aiyegbusi, O. L. et al. J. R. Soc. Med. https://doi.org/10.1177/ 01410768211032850 (2021)

7. Dennis, A. et al. BMJ Open 11, e048391 (2021).

8. Long Covid Research Collection (accessed 1 November 2021); https://www.scienceopen.com/collection/LongCOVID

9. Amin-Chowdhury, Z. \& Ladhani, S. N. Nat. Med. 27, 1129-1130 (2021).

10. Callard, F. \& Perego, E. Soc. Sci. Med. 268, 113426 (2021).

\section{Acknowledgements}

Some of the studies described in this correspondence were jointly supported by NIHR and UKRI. The views expressed in this article are those of the author(s) and not necessarily those of UKRI, NIHR or the Department of Health and Social Care. 
Author contributions

All authors conceived the idea for the article. A.R., L.O.M. and K.K. led on drafting, editing and revising the content, and A.R. and L.O.M. contributed equally as first authors. All other authors contributed to editing the content, and all authors approved the final version and are accountable for all aspects of this work.

Competing interests

M.C. is Director of the Birmingham Health Partners Centre for Regulatory Science and Innovation, Director of the Centre for Patient Reported Outcomes Research and is a NIHR Senior Investigator. M.C. receives funding from the NIHR Birmingham Biomedical Research Centre, the NIHR Surgical Reconstruction and Microbiology Research Centre and NIHR ARC West Midlands at the University of Birmingham and University Hospitals Birmingham NHS Foundation Trust, Health Data Research UK, Innovate UK (part of UKRI), Macmillan Cancer Support, UCB and GSK Pharma. M.C. has received personal fees from Astellas, Aparito Ltd, CIS Oncology, Takeda, Merck, Daiichi Sankyo, Glaukos, GSK and the Patient-Centered Outcomes Research Institute (PCORI) outside of the submitted work. N.C. has received funding from AstraZeneca to serve on Data Safety and Monitoring Committees for clinical trials. K.K. is a director of the University of Leicester Centre for Ethnic Health Research, trustee of the South Asian Health Foundation, and chair of the Ethnicity Subgroup of the Scientific Advisory Group for Emergencies (SAGE). P.E. is director of the MRC Centre for Environment and Health and the NIHR Health Protection Research Unit in Chemical and Radiation Threats and Hazards. P.E. is a foundation professor of the UK Dementia Research Institute at Imperial College, an Associate Director of Health Data Research UK (London), and leads the Informatics and Biobanking theme at the NIHR Imperial Biomedical Research Centre.

\section{Pseudonymization of healthcare data in South Korea}

To the Editor - In many parts of the world, there is a growing interest in how best to utilize healthcare data while providing adequate privacy safeguards. Achieving this balance is both a technical matter and a regulatory and procedural matter. Recent experiences in South Korea have emphasized the role of pseudonymized personal information and provide useful insights for the rest of the world.

Korea's main data privacy law, the Personal Information Protection Act (PIPA), has a generally similar structure to the EU's General Data Protection Regulation (GDPR). The PIPA has been construed to impose a strong requirement for consent ${ }^{1}$. In 2020, Korea amended the PIPA and introduced the concept of pseudonymized data $^{2}$. Under the amended Korean law, as in the GDPR ${ }^{3}$, pseudonymized data are still considered statutorily defined personal information. Unlike other types of personal information, however, pseudonymized data can be used without obtaining the data subjects' consent.

The use of pseudonymized data is limited to scientific research purposes, statistical purposes and archiving purposes in the public interest. The amended PIPA also introduced a scheme for the combination of pseudonymized data, which can be carried out by designated expert agencies ${ }^{4}$. Data combination could take place not only between datasets containing healthcare data but also between datasets containing healthcare data and other types of data.

With the PIPA amendment in place, Korea's Ministry of Health and Welfare, together with the Personal Information Protection Commission, issued a healthcare data guideline for utilizing healthcare data in January 2021. Separately, by late 2020,

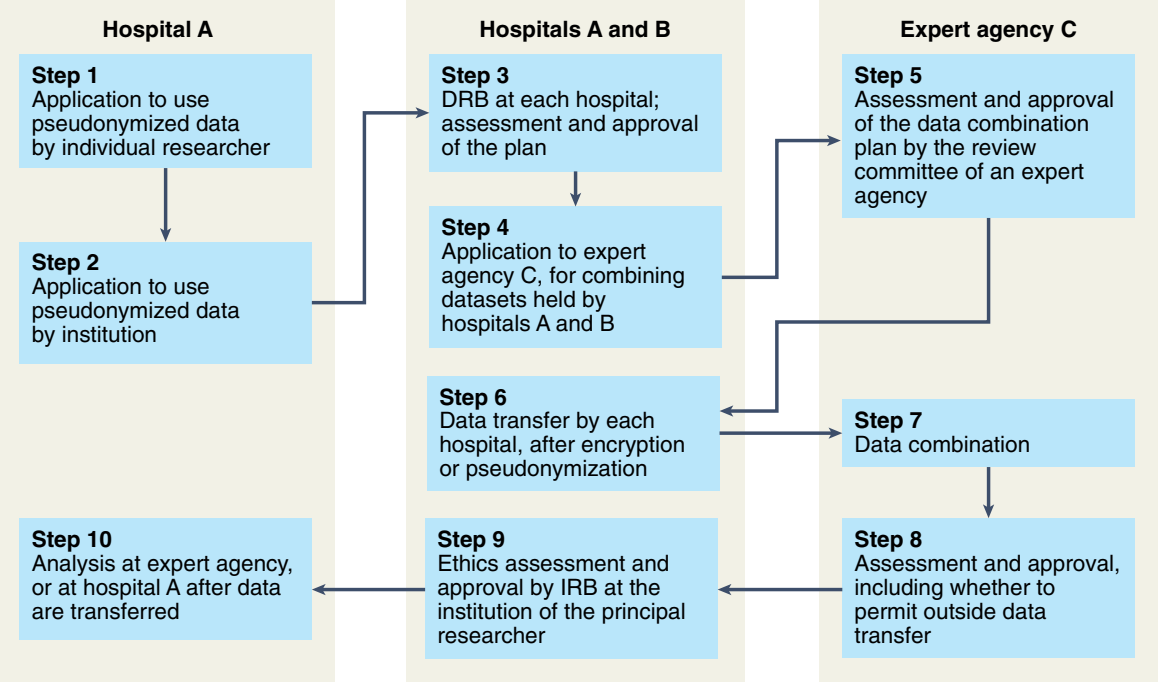

Fig. 1| An illustration of the utilization of pseudonymized and combined data. In this example, datasets held by two hospitals are pseudonymized and combined. Data combination takes place at an expert agency, and subsequent analysis can be conducted at this expert agency. If approved by the expert agency, the hospitals involved could extract the combined data to their research facilities for further analysis. IRB, institutional review board; DRB, data review board.

three agencies with expertise in healthcare data had been designated as expert agencies for the purpose of assessing the overall pseudonymization and data combination process, with more to be designated.

The Healthcare Data Guideline provides substantive as well as procedural safeguards applicable to the process of pseudonymizing healthcare data ${ }^{5}$. In terms of procedural safeguards, an important new concept was introduced: in order to utilize pseudonymized healthcare data, approval by a data review board (DRB) is now required. This is in addition to and separate from the existing requirement of institutional review board (IRB) approval. IRBs already have an existing mandate to review issues related to data privacy, and this mandate remains in place. The additional, mandatory $\mathrm{DRB}$ reflects the growing significance of considering privacy when utilizing healthcare data.

When pseudonymizing healthcare data, different statistical and technical measures are needed depending on the categories of individual data elements: i.e., depending on whether they are identifiers, quasi-identifiers or other attributes. For identifiers, the basic principle is to delete them or to substitute them with serial 